\title{
Large deviations and heterogeneities in a driven kinetically con- strained model
}

\author{
F. TurCI and E. PitARD \\ Laboratoire des Colloïdes, Verres et Nanomateriaux \\ Université Montpellier II and CNRS, 34095 Montpellier, France \\ PACS 05.60.-k - Transport processes \\ PACS 05.70.Fh - Phase transitions: general studies \\ PACS 05.70.Ln - Nonequilibrium and irreversible thermodynamics \\ PACS 64.70.Q- - Theory and modeling of the glass transition
}

\begin{abstract}
Kinetically Constrained Models (KCMs) have been widely studied in the context of glassy dynamics, focusing on the influence of dynamical constraints on the slowing down of the dynamics of a macroscopic system. In these models, it has been shown using the thermodynamic formalism for histories, that there is a coexistence between an active and an inactive phase. This coexistence can be described by a first-order transition, and a related discontinuity in the derivative of the large deviation function for the activity. We show that adding a driving field to a KCM model does not destroy this first-order transition for the activity. Moreover, a singularity is also found in the large deviation function of the current at large fields. We relate for the first time this property to microscopic structures, in particular the heterogeneous, intermittent dynamics of the particles, transient shear-banding and blocking walls. We describe both the shear-thinning and the shear-thickening regimes, and find that the behaviour of the current is well reproduced by a simple model.
\end{abstract}

Introduction. - Disordered materials, such as gels, glasses, complex fluids or granular materials, exhibit slow relaxation and heterogeneity, both in space and time. In particular, spatial dynamical heterogeneities, i.e the coex' istence of mobile particles and blocked particles, have been , widely studied [1] and have benefited from the results of KCMs (Kinetically Constrained Models), which by definition rely on specific dynamical rules depending on the mobility of the particles [2].

When driven by an external force, these complex systems exhibit specific spatial features, such as shearbanding [3. Another complication arises [4] with shearthickening, where viscosity increases when the driving force increases [5]. In this paper, we study a $\mathrm{KCM}$ driven by an external field, using both global observables (large deviation functions) and a microscopic description. This model, first introduced by Sellitto [6], has the advantage of displaying both shear-thinning and shear-thickening regimes: at small field, the current of particles is proportional to the field, whereas at large fields, the system has a negative differential resistance, i.e the current decreases. Similar effects have also been observed in electronic sys- tems [7] where negative differential conductivity can occur under strong electric fields.

In this context we have studied the thermodynamics of histories in the approach developed by Ruelle [8, 9] for deterministic dynamical systems. This formalism can be extended to Markov chains [10, and has been already developed for KCMs [11. In these latter studies, where the observable is the activity, one is able to probe trajectories in phase space with either finite, or almost zero activity, according to a "chaoticity temperature", $s$, which is introduced in the formalism. Contrarily to systems without kinetic constraints, there is a first-order transition at $s=0$ in the activity, which translates into a discontinuity of the derivative of the associated large deviation function. This transition is the global signature of the observation of dynamical heterogeneity. In the case of driven KCMs, we will focus on the large-deviation functions for both the activity and the current. We will also present a microscopic analysis of the structures involved in the dynamics, and relate them to global quantities.

Ruelle's formalism. - This formalism yields information about the fluctuations of temporal trajectories 
in configuration space, while the usual canonical thermodynamics approach yields information about the fluctuations associated with configurations. One defines a dynamical partition function, with a chaoticity temperature that is conjugated to the observable. Hence, if $O$ is a time-extensive observable, $Z_{O}(s, t)=\left\langle e^{-s O}\right\rangle$. The brackets mean that one is averaging over all possible histories between 0 and $t$. More precisely, $Z_{O}(s, t)=$ $\sum_{\text {histories }} \operatorname{Prob}$ (history) $e^{-s O \text { (history) }}$, where a history is the time series of configurations $\left\{C_{1}, C_{2}, \ldots, C_{t}\right\}$. In the long time limit, the partition function behaves like

$$
\lim _{t \rightarrow \infty} Z_{O}(s, t) \propto \exp \left\{t \psi_{O}(s)\right\}
$$

where $\psi_{O}$ is the large deviation function associated with the observable $O$. In practice, one is also interested by the mean of the observable in $s$-space, i.e, where the trajectories are biased by the factor $e^{-s O}$. This quantity is related to the large deviation function through the relation, in the large time limit: $\langle O(s, t)\rangle / t=-\psi_{O}^{\prime}(s)$.

The model. - In this paper, we study a two dimensional asymmetric simple exclusion process (ASEP) on a square lattice with dynamic constraints 6 . One can also view the model as the KA (Kob-Andersen) model in two dimensions -an example of KCM- [12, driven by an external field. The dynamical rule is such that a particle can move to a neighboring site only if it has at least two empty neighbours, before and after the move. Such a constraint mimics the fact that a particle in a dense disordered material can only move if it has enough free volume. Such a model always has a non-vanishing diffusion coefficient except for a density equal to 1 [13]. The motion of the particles is biased in one direction of the lattice by an external field $\vec{E}$, such that the probability for a particle to move in a certain direction is $p=\min \{1, \exp [-\vec{E} \cdot \vec{r}]\}$, where $\vec{r}$ indicates the displacement vector. The boundary conditions are periodic in both directions. In [6], the current was measured as a function of density $\rho$ and the field, showing several distinct behaviours. For densities below a threshold value (approximately $\rho_{c} \approx 0.79$ ) the system shows a monotonic growth of the current as a function of the external field (like in the non constrained ASEP model), which eventually saturates to a maximum current value. For $\rho \geq \rho_{c}$, the current has two different regimes: for $E$ small, a first ohmic regime (also referred to as a "shear thinning" regime) where the current grows proportionally to the field; and for $E$ large, a negative differential resistance regime (also called "shear thickening" regime) where the current decreases as the field increases. Such a behaviour has also been observed in other similar models of glasses [14. In this paper, we are mainly interested in the origin of the non-monotonic behaviour of the current as a function of the field. As a consequence we will consider only densities above the critical density $\rho_{c}$.

In the following, we will consider two different time extensive observables, namely the activity $K$ and the in-

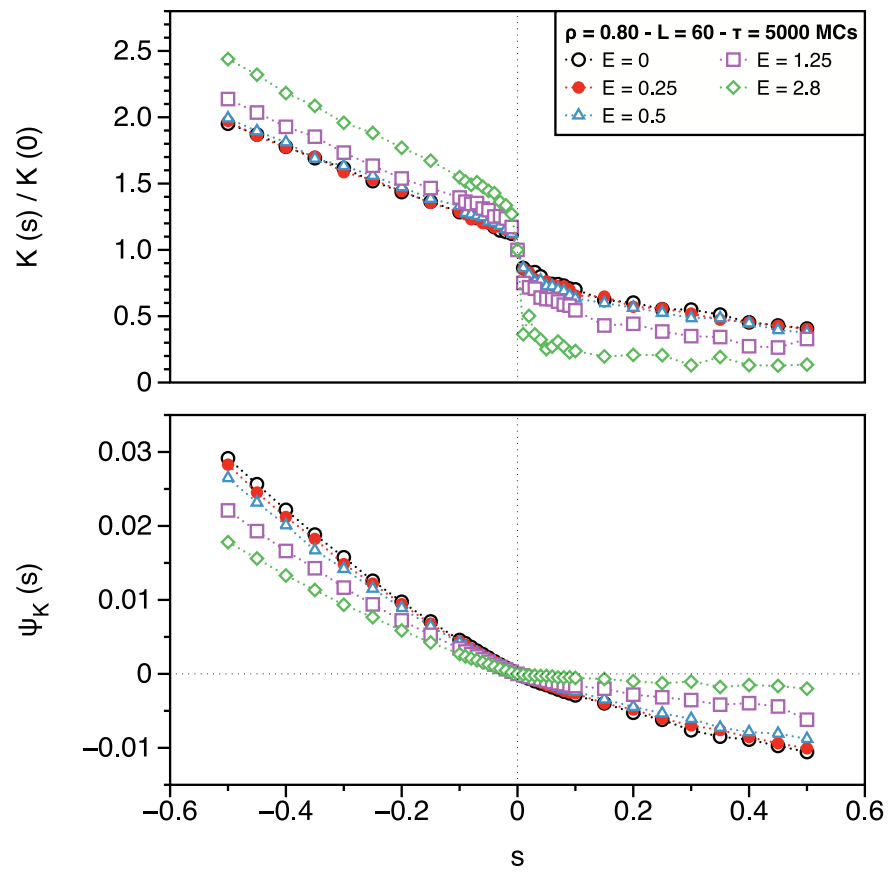

Fig. 1: The mean activity $K(s)$ in the modified dynamics, normalised by its value in $s=0$ (top), and its large deviation function $\psi_{K}(s)$ (bottom) for a system of size $L^{2}=60^{2}$, at high density $\rho=0.80$. For the simulation, we have considered 300 clones of the system evolving for a total time of $\tau=5000 \mathrm{MCs}$. For any value of the external field there is a discontinuity in the derivative of $\psi_{K}$ which corresponds to two different regimes in $K(s) / t$ : an active one $(s<0)$ and an inactive one $(s>0)$.

tegrated current $Q$. The activity $K(t)$ is defined as the total number of moves from time 0 to time $t$. The integrated current $Q(t)=\int_{0}^{t} J\left(t^{\prime}\right) d t^{\prime}$ is defined as the number of moves in the direction of the field from time 0 to time $t$. The current calculated in [6] is simply $Q(t) / t$.

In order to simulate the biased dynamics (the "sdynamics") and to compute the values of $K(s), Q(s)$ and their large deviations functions $\psi_{K}(s)$ and $\psi_{Q}(s)$, we have used the discrete time cloning algorithm proposed in reference 15, which was proved to be powerful enough for numerical studies of KCMs.

Large deviations computations. - The large deviation function for the activity has already been studied for different KCMs without driving such as the FredricksonAndersen Model, the triangular lattice gas model, the Kob-Andersen model [11. A first-order phase transition in $K$ was observed at $s=0$. Such a transition is the global signature for the coexistence at $s=0$ (which corresponds to the unbiased, physical system) of histories that have positive values of the activity (typical of $s<0$ ), and histories that have sub-extensive activity (typical of $s>0$ ). It is a translation in phase space of the dynamical heterogeneities one finds when looking at configurations. For non-driven models the activity's phase transition is 
Large deviations and heterogeneities in a driven kinetically constrained model


Fig. 2: The mean integrated current $Q(s)$ in the modified dynamics normalized by the value $Q(s=0)$ (top) and its large deviation function $\psi_{Q}(s)$ (bottom) plotted with a proper rescaling. The system has a size of $L^{2}=60^{2}$ sites and a density $\rho=0.8$. The maximum current phase is attained for a field $E^{*} \simeq 2$. Here we have considered 300 clones of the system evolving for $\tau=5000 \mathrm{MCs}$. The increase of the discontinuity of $Q(s)$ at $s=0$ is emphasized in the inset.

directly linked to the presence of kinetic constraints, since it disappears as soon as the constraints are removed. In our driven model, we find that this first-order transition still exists for the activity (see Fig. 1), and is by no way smeared out by the driving, whether the system is in the positive or in the negative differential resistance regime. The discontinuity observed at $s=0$ increases as $E$ gets larger. We checked that for all fields, the transition exists at the thermodynamic limit: we performed a finite-size study showing that the discontinuity is more and more visible as the size of the system increases. In order to do this study accurately, we needed more and more clones and longer simulation times for larger system sizes, in order for the cloning algorithm to converge.

We considered then the integrated current, flowing in the direction of the external field. It is linked to the activity since it is the difference between the activity in the positive direction of the field and the one in the negative direction. In the case of a non-constrained asymmetric exclusion process in one dimension, it has been shown 16] that the current's large deviation function is a regular convex function with a symmetry around $E / 2$ due to the fluctuation theorem [17. This means that no discontinuity exists for any value of $s$ for the derivative of $\psi_{Q}(s)$ in the non constrained case, or, in other terms, $Q(s)$ is a

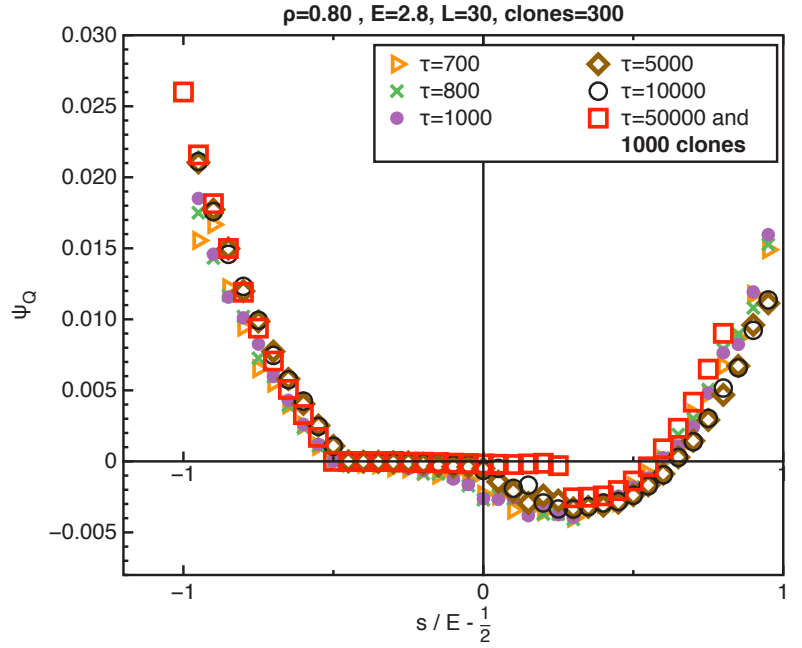

Fig. 3: The large deviation function $\psi_{Q}(s)$ for a system size of $L^{2}=30^{2}$ sites and a density $\rho=0.8$. Here we have considered 300 clones evolving for different times $\tau$. We also show the result for 1000 clones and a very long simulation time, suggesting the asymptotic symmetric shape, and a clear discontinuity of the derivative at $s=0$.

continuous function. We checked this in the case of the two dimensional ASEP. Moreover, a perfect collapse of the $Q(s) / t$ and $\psi_{Q}(s)$ curves can be found when rescaling $s$ by $s / E$ and the ordinates according to the scaling used in Fig. 2 ,

If we introduce the dynamical constraints we find (see Fig. 2) corrections to this perfect scaling. For small fields no discontinuity can be found (even if one uses more clones and longer times in the simulation); the large deviation function $\psi_{Q}(s)$ has the same shape we should expect for a non constrained model (2d-ASEP). Conversely, if we increase the field, a gap between the left and the right derivatives of $\psi_{Q}(s)$ is generated, and the size of the gap increases continuously as we increase the field. In the limit of large system sizes, the discontinuity in the derivative of $\psi_{Q}(s)$ corresponds to a step discontinuity in the biased current $Q(s)$; we also performed this finite-size study, and found that the discontinuity is sharper and sharper as the size increases. Here, the convergence of the cloning algorithm at fixed $L$ is rather slow. Figure 3 illustrates this point: as time and the number of clones increase, the transition is more and more visible. This figure also suggests that $\psi_{Q}(s)$ should asymptotically converge to a symmetric shape with a central part equal to zero; this is actually what is predicted by the fluctuation theorem [17] and was found in a mean-field model 20. This asymptotic curve could not be obtained here, due to numerical limitations: this difficulty was already raised by Sellitto [6] who also found deviations from the fluctuation theorem, even at very large times. 

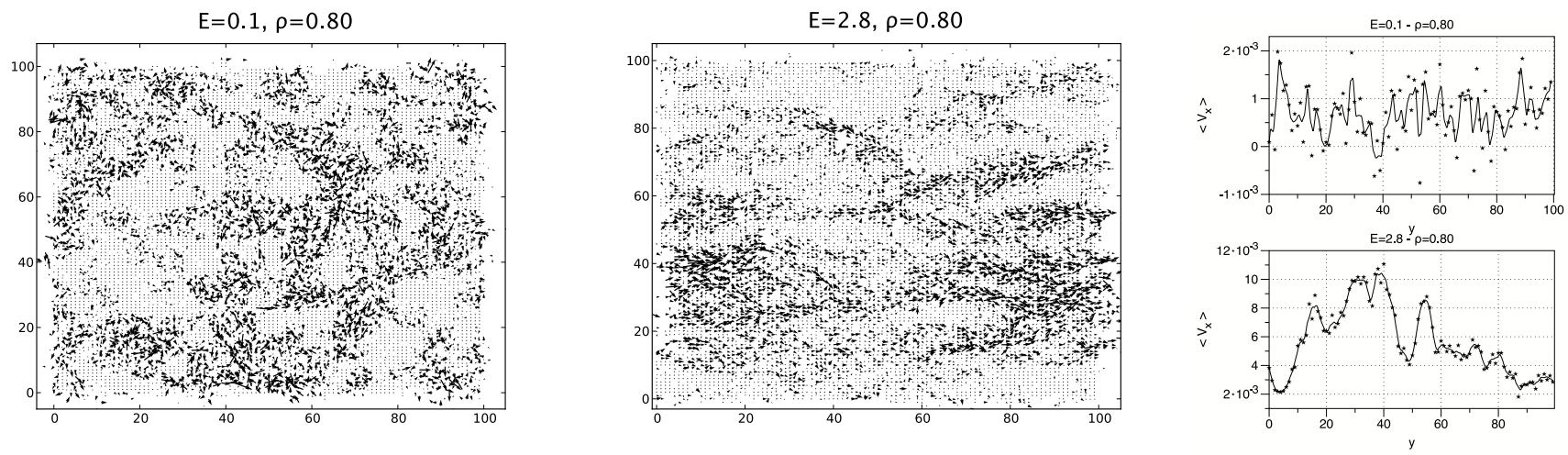

Fig. 4: On the left and middle: velocity field plots for two values of the external field, corresponding to the same value of the average current. The diffusive homogeneous behaviour at small fields can be contrasted with the localisation of transport in bands for large fields. On the right: the mean longitudinal velocity versus the direction transversal to the external field, for the same two values of the driving force.

Microscopic analysis. - Physically, this transition in $Q(s)$ and $\psi_{Q}(s)$ at $s=0$ corresponds to a coexistence between histories that have a positive current $(Q(s)$ is positive for $s<0$ ) from histories that have a subextensive current (for $s \rightarrow 0^{+}$we have no current, and nearly all particles are blocked).

In order to better investigate the emergence of the negative differential resistance behaviour, we have analyzed some microscopic aspects of the dynamics. These microscopic observations are also useful in order to understand the behaviour of the large-deviation functions described above, in the regimes of small and large forcing.

Simple observations of the dynamics of the system show that the driving by the field induces the formation of peculiar void structures, composed of quasi-linear segments of empty spaces, in the direction perpendicular to the field. The particles actually accumulate in some dense, blocked regions of the sample, separated by these "domain walls". These domain walls are hard to remove because of the kinetic constraint; their lifetime is long compared to the microscopic dynamics. In other words, the driving, combined with the dynamical restrictions, is at the origin of structures that slow down the dynamics. This effect is enhanced when the field is increased, yielding shearthickening behaviour. The domain walls can be directly visualized in the insets of figure 5 for small and large fields.

At the level of single particle displacements, the existence of such domains is also at the origin of different kinds of moves. For small fields (and small currents) the behaviour is almost diffusive, the particles randomly explore the region around their starting point, even coming back and forth on their own path. In the negative resistance regime (large fields) few particles manage to cover very long distances with almost ballistic trajectories, while others remain caged in the vicinity of their starting point. The moving particles actually diffuse in the vertical direction for some time before making some direct jump in the direction of the field. Such jumps actually correspond to the sudden disappearance of the empty domain walls. This behaviour is probably at the origin of superdiffusivity [6], which has also been observed in granular jammed systems [18. Moreover, it corresponds to a clear intermittent dynamics where the current is locally large for a short period of time.

We have computed the distribution of wall sizes $P(w)$ in the stationary regime. We have found that $P(w)$ is well approximated by a simple exponential in a large range of values of $\rho$ and $E$, allowing to define an average size $<w>(\rho, E)$. This average wall size grows as the density or the field grows, and saturates at large values of the external field [19. We can in fact rationalize the behaviour of the current, $J(E)$, by the following phenomenological argument. Let us assume that one can attribute the slowing down of the dynamics by the mere presence of the walls. The average current counts the number of particles susceptible to move in the direction of the field, and not blocked by the presence of a wall: $J(E)=A\left(1-e^{-E}\right)\left(1-p_{\text {blocked }}\right)$. We assume that the probability to be blocked is proportional to the number of blocked particles confined on the left border of a wall. To lowest order in the wall size we assume a linear dependence of $p_{\text {blocked }}$ on $\langle w\rangle(\rho, E)$ : $p_{\text {blocked }}=\alpha<w>(\rho, E)$, where $\alpha$ depends in principle on $\rho$ and $E$. We found that the curves for $J(E)$ could be very well fitted by this formula, taking $\alpha$ independent of $E$ (see Fig. 5). The parameter $\alpha$, which can be shown in a more refined study [19] to depend on microscopic geometric details, such as the number of walls or the average distance between them, is found to be very weakly dependent on $\rho$ in the range studied. The assumptions made here can explain the fact that the fit actually begins to fail at large $\rho$ : then $\langle w\rangle$ gets larger, $P(w)$ starts to deviate from an exponential form, showing that the appropriate expression for $p_{\text {blocked }}$ may change.

At the level of the entire sample, we have drawn the 
velocity field plot for the small and large values of the driving force, from which one can obtain the velocity profile for the longitudinal component of the speed vector (see fig. (4). Velocities in these plots are defined over a time lag of the order of the relaxation time; they also correspond to displacement snapshots on this timescale. These plots show that at large fields the system gets more and more organized, with bands of moving particles coexisting with static regions blocked by walls, reminiscent of shear banding observed in complex flows. This illustrates the first-order transition for the large deviation of the current at large fields. The high velocity bands have a macroscopic size and involve the whole longitudinal length of the system. At small field, the current is more homogeneous, which is consistent with the absence of transition in the large-deviation function for the current.

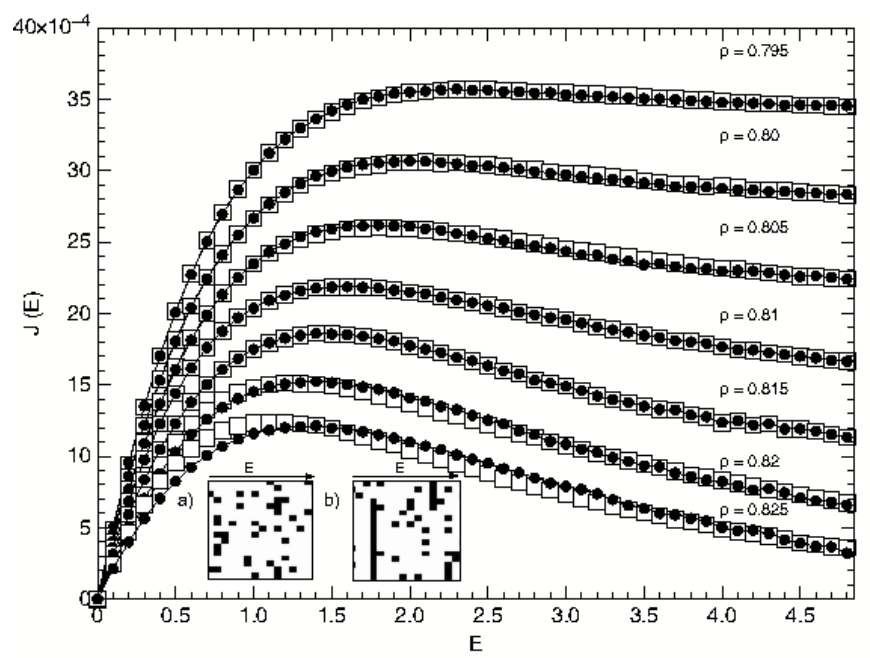

Fig. 5: Simulated flow curves (white squares) fitted by the phenomenological model $J(E)=A\left(1-e^{-E}\right)(1-\alpha<w>)$ (black dots).Densities range from 0.795 to $0.825, L=100$, and $\langle w\rangle$ is determined independently from the simulation. Below are shown two fractions of snapshots of configurations taken at $\rho=0.82$, a) $\mathrm{E}=0, \mathrm{~b}) \mathrm{E}=5$, illustrating the presence of walls. The empty sites are in black, the particles in white: the empty sites organize into vertical walls at large fields.

Conclusion. - We have found that the coexistence of mobile and blocked trajectories in configuration space is a conserved feature in KCMs even in the presence of external driving. This is illustrated quantitatively through the study of the large deviations for the activity and for the current. In the shear-thinning regime, the microscopic behaviour is reminiscent of the dynamical heterogeneities found in $\mathrm{KCMs}$, coming from dynamical restrictions; there is a transition in the large deviation function for the activity, but not for the current. In the shear-thickening regime, the field induces a structuration of the flow, with domain walls separating dense jammed regions, as well as transient shear bands. The discontinuity found at large fields in $\psi_{Q}^{\prime}(s=0)$ illustrates the dominant role of the blocking walls in the structuration of the displacement field. We finally note that we have very recently found a preprint by Speck and Garrahan on a related subject, where a firstorder transition is also found for the entropy production for driven KCMs [20].

$$
* * *
$$

We are grateful to V. Lecomte, F. van Wijland, J. Kurchan, L. Berthier for interesting discussions, and to M. Sellitto for correspondance. FT is supported by the French Ministry of Research and EP by CNRS and PHC no 19404QJ.

\section{REFERENCES}

[1] Sillescu H., J. Non-Cryst. Solids, 243 (1999) 81. Ediger M.D., Annu. Rev. Phys. Chem., 51 (2000) 99. Glotzer S.C., J. Non-Cryst. Solids, 274 (2000) 342. Richert R., J. Phys. Condens. Matter, 14 (2002) R703. Andersen H.C., Proc. Natl. Acad. Sci. U. S. A., 102 (2005) 6686.

[2] Ritort F. and Sollich P., Adv. Phys., 52 (2003) 219.

[3] Losert W., Bocquet L., Lubensky T.C. and Gollub J.P., Phys. Rev. Lett., 85 (2000) 1428.

Fielding F.M., Cates M.E. and Sollich P., Soft Matter, 5 (2009) 2378.

[4] Liu C.-H. and Pine D., Phys. Rev. Lett., 77 (1996) 2121. Bertrand E., Bibette J. and Schmitt V., Phys. Rev. $E, 66$ (2002) 060401(R).

Fall A., Huang N., Bertrand F. Ovarlez G. and Bonn D., Phys. Rev. Lett., 100 (2008) 018301.

[5] Larson R.G., The Structure and Rheology of Complex Fluids (Oxford University Press, Oxford) 1999.

[6] Sellitto M., Phys. Rev. Lett., 101 (2008) 048301.

Sellito M., Phys. Rev. E, 80 (2009) 011134.

[7] Levin E.I. and Shklovski B.I., Solid State Commun., 67 (1988) 233.

Nenashev A.V. ET AL, Phys. Rev. B, 78 (2008) 165207.

[8] Ruelle D., Thermodynamic Formalism (Addison-Wesley, Reading) 1978.

Eckmann J.-P. and Ruelle D., Rev. Mod. Phys., 57 (1985) 617.

GASPARD P., Chaos, scattering and statistical mechanics (Cambridge University Press, Cambridge) 1998.

[9] Touchette H., Phys. Rep., 478 (2009) 1.

[10] Lecomte V., Appert-Rolland and van Wijmand F., Phys. Rev. Lett., 95 (2005) 010601.

[11] Garrahan J.P., Jack R.L., Lecomte V., Pitard E., van DuijvendiJk K. and van Wijland F., Phys. Rev. Lett., 98 (2007) 195702.

Garrahan J.P., Jack R.L., Lecomte V., Pitard E., van Duijvendijk K. and van Wijland F., J. Phys. A, 42 (2009) 075007.

[12] Kob W. and Andersen H.C., Phys. Rev. E, 48 (1993) 4364.

[13] Toninelli C., Biroli G. and Fisher D., Phys. Rev. Lett., 92 (2004) 185504.

Marinari E. and Pitard E., Europhys. Lett., 69 (2005) 235. 
[14] Jack R.,Kelsey D., Garrahan J.P. and Chandler D., Phys. Rev. E, 78 (2008) 11506.

[15] Giardinà C., Kurchan J. and Peliti L., Phys. Rev. Lett., 96 (2006) 120603.

[16] Bodineau T. and Derrida B., Phys. Rev. E, 72 (2005) 066110.

Lecomte V., PhD thesis, 2007 (.)

[17] Lebowitz J.L. and Spohn H., J. Stat. Phys., 95 (1999) 333.

[18] Lechenault F., Dauchot O., Biroli G. and Bouchaud J.P., Europhys. Lett, 83 (2008) 46003.

[19] Turci F. and Pitard E., in preparation.

[20] Speck T. and Garrahan J.P., arXiv:1004.2698 